\title{
1 Genomic expansion of archaeal lineages resolved from deep Costa Rica sediments
}

2 Ibrahim F. Farag ${ }^{1}$, Jennifer F. Biddle ${ }^{1}$, Rui Zhao ${ }^{1}$, Amanda J. Martino ${ }^{2}$, Christopher H. House ${ }^{3}$,

3 Rosa I. León-Zayas ${ }^{1,4}$

$4 \quad{ }^{1}$ School of Marine Science and Policy, University of Delaware, Lewes DE 19968

$5 \quad{ }^{2}$ Department of Biology, St. Francis University, Loretto PA 15940

$6 \quad{ }^{3}$ Department of Geosciences, Pennsylvania State University, University Park PA 16802

$7 \quad{ }^{4}$ Department of Biology, Willamette University, Salem OR 97301

\section{Abstract}

10 Numerous archaeal lineages are known to inhabit marine subsurface sediments, although their

11 distributions, metabolic capacities and interspecies interactions are still not well understood.

12 Abundant and diverse archaea were recently reported in Costa Rica (CR) margin subseafloor

13 sediments recovered during IODP Expedition 334. Here, we recover metagenome-assembled

14 genomes (MAGs) of archaea from the CR-margin and compare them to their relatives

15 from shallower settings. We describe 31 MAGs of 6 different archaeal lineages (Lokiarchaeota,

16 Thorarchaeota, Heimdallarchaeota, Bathyarcheota, Thermoplasmatales and Hadesarchaea) and

17 thoroughly analyze representative MAGs from the phyla Lokiarchaeota and Bathyarchaeota.

18 Our analysis suggests the potential capabilities of Lokiarchaeota members to anaerobically

19 degrade aliphatic and aromatic hydrocarbons. We show it is genetically possible and

20 energetically feasible for Lokiarchaeota to degrade benzoate if they associate with organisms

21 using nitrate, nitrite and sulfite as electron acceptors, which suggests a possibility of syntrophic

22 relationships between Lokiarchaeota and nitrite and sulfite reducers. The novel Bathyarchaeota

23 lineage possesses an incomplete methanogenesis pathway lacking the methyl co-enzyme M 
24 reductase complex and encodes a non-canonical acetogenic pathway potentially coupling methylotrophy to acetogenesis via the methyl branch of Wood-Ljundahl pathway. These novel metabolic characteristics suggest the potential of this Bathyarchaeota lineage to be a transition

27 between methanogenic and acetogenic Bathyarchaeota lineages. This work substantially expands

28 our knowledge about the metabolic function repertoire of marine benthic archaea.

Introduction

Marine subsurface sediments are full of diverse archaeal lineages[1], although their

31 distributions, ecological roles and adaptation strategies are still not well understood[2][3][4][5].

32 Metagenomic sequencing and single cell genomics have enabled the discovery of a great

33 number of organisms, the elucidation of new metabolisms, the expansion of known lineages and

34 the redefinition of portions of the tree of life[6][7], [8][9][10][11][12][13][14]. However, there is

35 still a paucity of genomes resolved from the deep marine subsurface, meaning the niche specific

36 adaptations in deep biosphere is not yet well understood.

Recently, the tree of life has been greatly expanded with the discovery of the Asgard superphylum, a deeply-branching monophyletic group thought to be some of the closest relatives to the eukaryotic branch of life[11][15]. Genome analyses of Asgard archaea have suggested diverse metabolic functions extending from an autotrophic lifestyle, primarily dependent on

41 carbon fixation via Wood-Ljundahl pathway and acetogenesis, to a heterorganotrophic lifestyle

42 consuming proteins and aliphatic hydrocarbons, using methyl-CoM reductase-like enzymes, to

43 recycle aliphatic hydrocarbons released from the subsurface[16][17][18]. Asgard members were

44 proposed to be engaged in symbiotic partnerships involving syntrophic transfers of hydrogen and

45 electrons following the 'reverse flow model' [16]. 
Members of another archaeal phylum in the deep subsurface, Bathyarchaeota, are

47 characterized by their wide metabolic repertoire enabling heterotrophic scavenging of proteins, carbohydrates, short chain lipids and other reduced compounds as substrates as well as their methane-metabolizing potential [19]. However, bathyarchaeotal genomes have also suggested the potential for carbon fixation and acetogenesis [20]. The evolutionary path describing the acquisition of both methanogenesis and acetogenesis pathways in Bathyarchaeota remains unresolved [20][21]. International Ocean Discovery Program (IODP) Expedition 334, was recently shown to host abundant archaea [22]. Here we examine in detail the archaeal genomes recovered from the

56 Costa Rica Margin and compare them to their relatives recovered from shallower sites. In this study, we report 31 archaeal metagenome-assembled genomes (MAGs) belonging to six different archaeal lineages (Lokiarchaeota, Thorarchaeota, Heimdallarchaeota, Bathyarcheota, analyze representative MAGs of two novel archaeal lineages belonging to phyla Lokiarchaeota

61 and Bathyarchaeota. Our analysis suggests that Lokiarchaeota genomes encode for genes

62 dedicated to process and degrade aliphatic and aromatic hydrocarbon anaerobically. We also

63 describe a metabolically novel Bathyarchaeota, which lacks methyl co-enzyme M reductase

64 (MCR) complex and possesses a non-canonical acetogenic pathway linking methylotrophy to

65 acetogenesis via the methyl branch of Wood-Ljundahl pathway. Lastly, we integrate genomic and thermodynamic modeling to underline the ecological and physiological conditions that could

67 drive the syntrophic interactions among CR-Asgards and the development of non-canonical 
Results

\section{Overall archaeal abundance and community structure}

High abundances of archaea across sediment samples collected from five depths located representing 3\%) (Figure S1).

We compared two million raw metagenome reads (150 bp) for each dataset to the NCBI respective phylogenetic group at the phylum level, while the phylogenetic signatures were not clear in the remaining reads $(92-94 \%)$. The percentage of total prokaryote reads belonging to archaea ranged from 5\% (at 93 mbsf, 1378) to 26\% (at 32 mbsf, 1378).. Overall, community

86 structure composition analysis conducted on metagenomic raw reads indicated the prevalence of

87 two archaeal phyla in the Costa Rica margin: Lokiarchaeota and Bathyarchaeota (Figure 1B).

88 Lokiarchaeota were most abundant among the archaea reads at the $32 \mathrm{mbsf}$ samples in core

89 1378, while Bathyarchaeota reads were most abundant at 22 mbsf and 45 mbsf in core 1379. 


\section{General genomic features of the abundant archaeal lineages}

Across the five-depths analyzed, 31 different draft archaeal MAGs were recovered (Table

94 1). Genomic analyses were only performed on the 11 MAGs showing high completeness and low

95 contamination percentages (above 60\% completeness and below 5\% contamination). Overall,

96 completeness varied from $32 \%$ to $99 \%$ with an average of $50 \%$ and contamination varied from 0

97 to $10 \%$ with an average of $8 \%$ (Table 1 ). Genome qualities were further assessed by comparing

98 their predicted proteins against the NCBI (nr) database to evaluate the extent of phylogenetic

99 consensus within the binned genomes (Figure 2A). Overall, the taxonomic affiliations of the

100 majority of the predicted proteins (60-75\%) in each genome agreed with their respective

101 phylogenetic group, except CR-12 in which only $15 \%$ of the encoded proteins assigned to

102 Heimdallarchaeota and was excluded from further analysis.

104 proteins (Figure 1A and Figure S2)[12]. The recovered MAGs were affiliated to six different

105 phylogenetic lineages, namely, Lokiarchaeota, Thorarchaeota, Heimdallarchaeota,

106 Bathyarcheota, Thermoplasmatales and Hadesarchaea. 
(A)
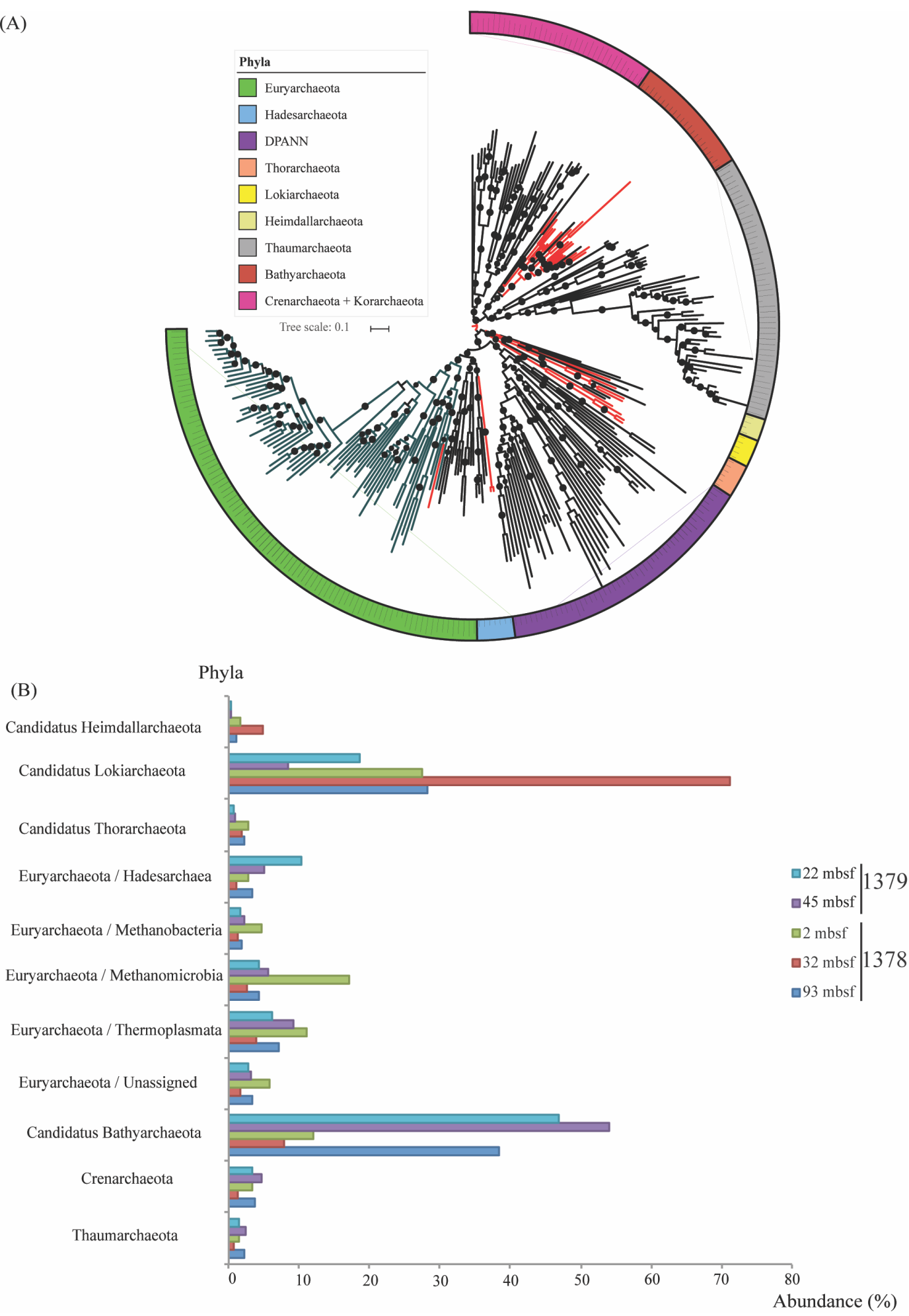

Figure 1. (A) Phylogenetic placement of the Costa Rica archaeal draft genomes (genomes of this study are

109 highlighted in red). The maximum-likelihood phylogenetic tree was calculated based on the concatenation of 16

110 ribosomal proteins (L2, L3, L4, L5, L6, L14, L15, L16, L18, L22, L24, S3, S8, S10, S17, and S19) retrieved from 
111 the Costa Rica archaeal genomes and 231 reference archaeal genomes representing 13 different archaeal phyla. The

112 relationships were inferred using the best fit substitution model (VT+F+R10) and nodes with bootstrap support

$113>80 \%$ were marked by black circles. Scale bar indicates substitutions per site. The tree is available with full

114 bootstrap values in Newick format in the Supplementary Data.

115 (B) Relative abundance percentages of all archaeal lineages making up $>\mathbf{1 \%}$ of the total communities. This

116 graph was calculated by parsing the raw reads against NCBI (nr) database applying e-value cut off score 1e-5.

117 Distribution of Eukaryotic signature protein (ESP) homologs in Costa Rica archaea MAGs

All archaeal MAGs recovered from Costa Rica sediments encoded eukaryotic signature

119 proteins (ESPs). These ESPs include protein homologs dedicated for information processing,

120 trafficking machineries, ubiquitin system, cell division and cytoskeleton formation. The Asgard

121 archaea genomes recovered from CR, e.g. Heimdallarchaeota and Lokiarchaeota MAGs

122 (CR_06, CR_11), showed significantly higher numbers of eukaryotic homologs and covered

123 broader classes of ESPs (Figure 2b). However, ESPs were also detected in the Bathyarchaeota,

124 Hadesarchaeota and Thermoplasmata MAGs recovered from CR (Figure 2b). Yet, only the

125 Asgard genomes have the homologs for cell division and cytoskeleton.
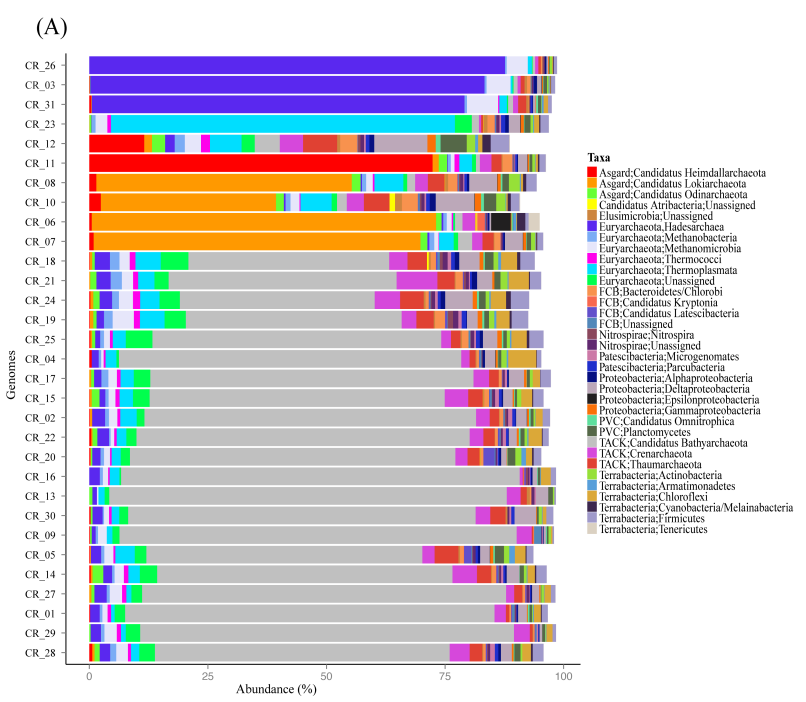

(B)

127 Figure 2. (A) Phylogenetic distributions of the predicted proteins encoded by the archaeal MAGs, the

128 phylogenetic assignments were performed through comparing the proteins to the NCBI (nr) proteins 

patterns of eukaryotic homologs in the recovered archaea MAGs. The color along the left side shows the completeness of the genome bin, as less complete bins would be expected to contain fewer homologs. Only the

132 Asgard archaea contain cell division/cytoskeleton homologs. degradation of hydrocarbon compounds (Figure 3A) that are abundant in Costa Rica (CR) margin [24]. MCR complex genes ( $m c r A B C D G)$ were completely absent from both the MAGs

138 and the entire metagenomes, which indicates that short chain alkanes are not oxidized using

139 MCR complex in CR sediments. All metagenomic reads and MAGs were screened for possible

140 alternative hydrocarbon degradation pathways using custom HMM searches specifically

141 targeting key metabolic genes for aliphatic and aromatic hydrocarbons degradation pathways

142 (Figure 3A). Multiple pathways were successfully identified including glycyl-radical enzymes

143 (GREs) related genes coupled with n-alkane succinate synthase (AssA) and benzylsuccinate 144 synthase (BssA), which activates $n$-alkanes and mono-aromatic compounds, respectively, by

145 forming C-C bond between these compounds and fumarate to form hydrocarbon adducts

$146[25][26]$. Benzylsuccinate synthesis is the initial step for aromatic hydrocarbon mineralization, in

147 which benzylsuccinate is converted to benzoyl-CoA[25]. Interestingly, the capability of ATP-

148 dependent Benzoyl-CoA reductase (BCR) complex utilization was identified in some CR Asgard

149 members (Lokiarchaeota, Thorarchaeota and Heimdallarachaeota) (Figure 3). This reaction

150 can dearomatize the benzoyl-CoA to dienoyl-CoAs as the first step in aromatic hydrocarbon

151 degradation and then couple this reaction with a beta-oxidation pathway to ultimately produce

152 acetyl-CoA, similar to the mechanism previously reported in the denitrifying bacteria Thauera 
153 aromatica [27]. Since Lokiarchaeota CR_06 had the least contamination levels $<2 \%$, we used

154 this MAG to verify the presence of aromatic hydrocarbon degradation function mediated by

155 BCR complex in Asgards. Phylogenetic analysis of the BCR subunit B recovered from

156 Lokiarchaeota CR_06 showed their affiliation to class Bzd, which is composed of four subunits

157 (BzdONPQ) (Figure 3C). This BCR type was originally discovered in Betaproteobacteria,

158 Azoarcus evansii and the ones detected in CR_06 is closely related to BCRs detected in different

$159 \beta, \delta$ proteobacteria, and other archaeal lineages (e.g. Lokiarchaeota, Bathyarchaeota and

160 Archaeoglobus) (Figure 3C)[28]. Subunits P and Q have ATP binding/ATPase functional

161 domains and are linked together by a [4Fe-4S] cluster. Reduced ferrodoxins transfer electrons to

162 the CoA- ester-binding domains (subunits $\mathrm{O}$ and $\mathrm{N}$ ), which catalyzes the cleavage of benzoyl-

163 CoA aromatic ring and yields dienoyl CoA product (Figure 3A) [28]. In CR_06, the four

164 subunits of BCR complex were located in one contiguous operon (CR_06- contig-100_3495),

165 however due to the high fragmentation of the genomes no reliable phylomarker genes were

166 found in the adjoining genomic neighborhoods. A plausible explanation for the high

167 fragmentation levels of the CR_06 (number of scaffolds=1110), even though the genome

168 exhibited high coverage levels $>200 x$, is that there are high levels of intra-lineage strain

169 heterogeneity. This is confirmed by the ANI values (ANI $=70-80 \%$ ) between MAGs of the same

170 phylogenetic group and disparities in their coverage levels (table 1 and table S3).

172 Lokiarchaeota CR_06 were confirmed by the following observations: 1) contigs encoding for

173 the four subunits of BCR complex fall within the same coverage and GC\% range of the rest of

174 the contigs in the same MAG (Figure S3); 2) phylogenetic trees of BzdO and Q protein

175 sequences detected in the CR_06 were placed as siblings to BCR sequences belonging to 

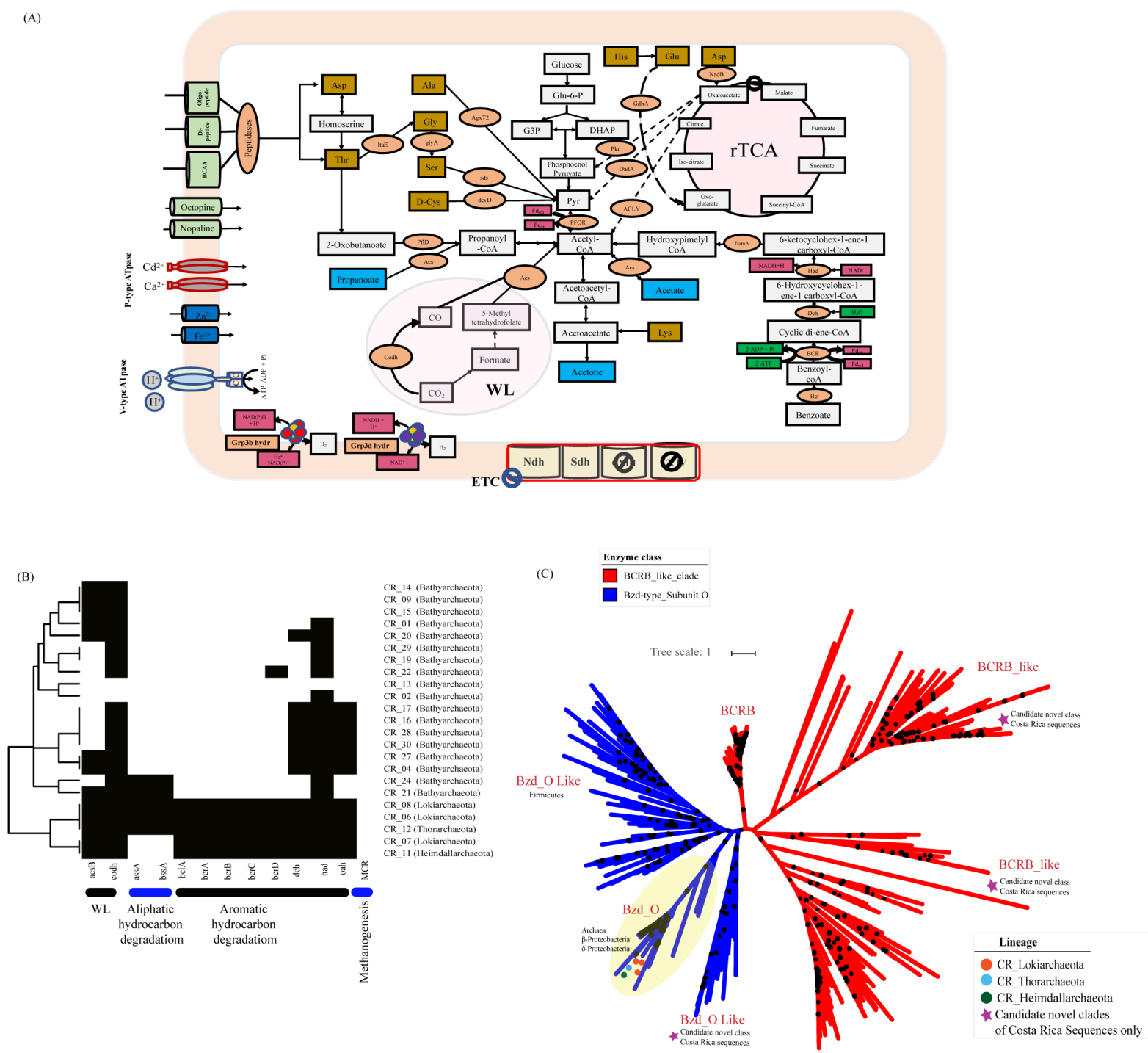

181 Figure 3. (A) Metabolic reconstruction of the CR_Lokiarchaeota bin (CR_06). Central metabolic pathways

182 are shown in gray boxes, carbon fixation pathways (WL and rTCA cycles) are shown in pink, electron transport

183 chain (ETC) proteins are shown in yellow, fermentation products are shown in blue boxes, amino acids are shown in

184 brown boxes, enzymes and enzyme complexes are shown in orange circles, energy carriers are shown in red, energy 
molecules are shown in dark green, metabolite and amino acid transporters are shown in light green, and cation

transporters are shown in dark blue. (B) Distribution patterns of the hydrocarbon degradation pathways among

187 the constructed CR-Archaea genomes. Genomes were clustered based on presence (black) /absence (white)

188 profiles using Euclidean distance and average linkage method. X axis represents enzymes included in the analysis,

189 bottom bars are the pathways that these enzymes represent and y axis includes bin names/phylogenetic affiliations.

190 (C) Maximum likelihood tree of the benzoyl-CoA reductase subunit B. The tree was calculated using the best fit

191 substitution model $(\mathrm{VT}+\mathrm{F}+\mathrm{R} 7)$ that describes the evolutionary relationships between $\mathrm{BCRB}$ families. The tree was

192 made using reference sequences under the KEGG entry (K04113) collected from AnnoTree[30] and branch location

193 was tested using 1000 ultrafast bootstraps and approximate Bayesian computation, branches with bootstrap support

$194>80 \%$ were marked by black circles. Blue and Red clades highlight sequences belong to Bzd_O and BCR_B

195 subfamilies, respectively. Scale bar indicates substitutions per site. Sequences from CR_Lokiarchaeota bins was

196 marked with red circles, CR_Thorarchaeota bin was marked with light blue and CR_Heimdallarchaeota was

197 marked with green. Candidate novel clades present in the Costa Rica metagenomic datasets were marked with

198 purple stars. The tree is available with full bootstrap values in Newick format in the Supplementary Data.

\section{Fate of the degraded aromatic hydrocarbons and syntrophic interactions}

200 CR_Lokiarchaeota (CR_06) as well as other Asgard in the CR margin could potentially grow

201 heterotrophically on aromatic hydrocarbons. Though, the absence of genes encoding for different

202 types of cytochrome oxidase and anaerobic respiration from the genome content of the Asgard

203 MAGs (CR_06, 07, 08, 11 and 12) indicate their inability to completely mineralize these

204 hydrocarbons to $\mathrm{CO}_{2}$ and $\mathrm{H}_{2} \mathrm{O}$, which would allow a high energy yield. They encode for the

205 genes mediating the fermentation of these organic macromolecules to acetate and other reduced

206 products, which is thermodynamically unfavorable under CR conditions with a positive $\Delta \mathrm{rG}^{\prime}$

207 value $\left(\Delta \mathrm{rG}^{\prime}=196.3[\mathrm{~kJ} / \mathrm{mol}]\right)$ (Figure 3 and table $\left.\mathrm{S} 1\right)$, suggesting that these genes are maintained

208 due to a thermodynamically favorable force. Due to the genome incompleteness ( $86 \%$ complete),

209 we cannot rule out the possibility for the presence of complete aromatic hydrocarbon 
210 mineralization pathways using one or more of oxidized substrates as electron sinks (Table 1).

211 More likely, however, Lokiarchaetoa are gaining energy through syntrophic interactions with

212 partners capable of oxidizing the biodegradation intermediates.

213 Here, we inferred the identity of the potential syntrophic partners under marine subsurface

214 conditions by comparing all the possible metabolic and thermodynamic scenarios, gauging each

215 scenario based on the presence of the metabolic pathways in our metagenomic datasets and the

216 thermodynamic feasibility under each condition (Figure 3B, Table S1 and S6). We calculated the

217 Gibbs free energy of coupled reactions under a wide range of substrate concentration conditions:

218 Reaction 1-5 (Table S1, Figure S5). Best conditions suggest that the degradation of benzoate (the

219 central metabolic intermediate in aromatic hydrocarbon degradation pathways) potentially occurs

220 under the following metabolic conditions ( $\mathrm{a}, \mathrm{b}$, and c): (a) benzoate mineralization to $\mathrm{CO}_{2}$ and

$221 \mathrm{H}_{2} \mathrm{O}$ coupled with nitrite reduction to ammonia $\left(\Delta \mathrm{rG}^{\prime}=-1206.3[\mathrm{~kJ} / \mathrm{mol}]\right) ;(\mathrm{b})$ benzoate

222 mineralization to $\mathrm{CO}_{2}$ and $\mathrm{H}_{2} \mathrm{O}$ coupled with sulfite reduction to hydrogen sulfide $\left(\Delta \mathrm{rG}^{\prime}=-373.6\right.$

$223[\mathrm{~kJ} / \mathrm{mol}])$; and (c) benzoate mineralization to $\mathrm{CO}_{2}$ and $\mathrm{H}_{2} \mathrm{O}$ coupled with nitrate reduction to

224 nitrite $\left(\Delta \mathrm{rG}^{\prime}=-119.9[\mathrm{~kJ} / \mathrm{mol}]\right)$.

The type and complexity of the exchanged substrates are another key factor that may

226 shape the syntrophic relationship and the identity of the syntrophic partners of Lokiarchaeota..

227 The presence of genes encoding for membrane bound electron bifurcating classes of [NiFe]

228 hydrogenases, groups $3 \mathrm{~b}$ and $3 \mathrm{~d}$, which couple the oxidation of $\mathrm{NADH}^{+}$and $\mathrm{NADPH}^{+}$with $\mathrm{H}_{2}$

229 evolution (Figure S6)[31] also suggests there is syntrophic exchange of hydrogen between

230 Asgard and their partners. Additionally, we located genes encoding for $\beta$-oxidation enzymes

231 (enoyl-CoA hydratase, acyl CoA dehydrogenase, and acetyl CoA acetyl transferase) and various

232 fermentation pathways (acetate and formate) ) in Lokiarchaeota CR_06, which suggests that 
233 short chain fatty acid and different fermentation products could also be syntrophically exchanged

234 (Table S4). The diverse nature of substrates could facilitate the interactions between a broader

235 range of partners of diverse metabolic capabilities and support the conclusions driven from our

236 thermodynamic calculations.

237 It is worth noting that efficient substrate and electron exchange between syntrophic partners

238 require the presence of either biological conduits (e.g. type IV pili or flagella) or some sort of

239 electron shuttles allowing extracellular electron transfers (e.g. multiheme cytochromes) [32][33].

240 Hence, we screened CR_06 for these mechanisms and identified two candidate mechanisms for

241 interspecies substrate and electron exchange. First, flagellar proteins are encoded by the CR_06,

242 suggesting flagella as a potential structure mediating inter-species interactions. Second, CR_06

243 harbors a gene encoding for an oxidoreductase belonging to electron transfer flavoprotein-

244 quinone oxidoreductase (CR_06_contig-100_4953_2), ETF-QO/FixC family, which potentially

245 mediating the transfer of electrons across membranes.

Other metabolic features of Lokiarchaeota (CR_06)

The genomic analysis of Lokiarchaeota (CR_06) MAG also suggests versatile catabolic

248 capacities potentially targeting detrital proteins and short chain fatty acids (e.g. propanoate,

249 butyrate), which are abundant in benthic marine sediments. CR_06 MAG has a relatively large

250 number of peptidases encoding genes (92 peptidases/1Mbp) with diverse catalytic residues (e.g.

251 aspartic, metallo, serine, etc.), which potentially degrade detrital proteins (Figure S7). It also

252 contains genes encoding for various classes of facilitated and active transporters, which are

253 dedicated to shuttle oligo/di-peptides and single amino acids (e.g. polar and branched chain

254 amino acids) across the cell membrane. Also, CR_06 encoded for enzymes enabling the 
255 utilization of wide range of amino acids (e.g. aspartate, threonine, alanine, glycine, serine,

256 cysteine, histidine, glutamine, and lysine), channel them to the central metabolic pathways and

257 ultimately produce energy via fermentation (Figure 3A).

CR_06 MAG suggested the capacity to break down short chain fatty acids e.g.

propanoate, oxo-butanoate as other potential substrates. Both propanoate and oxo-butanoate are

260 converted to propanoyl CoA via formate acetyl transferase and acetyl synthase, respectively.

261 Then, the resulting propanoyl CoA is converted to acetyl-CoA via malonyl-CoA pathway.

262 CR_06 as well as other Asgards showed different autotrophic capacities enabling carbon fixation

263 to complex organic carbon compounds using both the Wood-Ljundahl (WL) pathway and the

264 reverse tricaboxylic acid (rTCA) cycle (Figure 3A).

\section{Metabolic features of CR-Bathyarchaeota}

The other main lineage of Archaea in these sediments is Bathyarchaeota. Bathyarchaeota

CR_14 is described in details since it has the highest quality at $>95 \%$ complete and $4 \%$

268 contaminated. Phylogenomic analysis of Bathyarchaeota MAGS showed that CR_14 is clustered

269 together with other CR_Bathyarcaheota in two distinct clades within Bathyarchaeota phylum

270 (Figure S2). Also, relatively low similarity scores were observed between CR_14 and other

271 reference Bathyarchaeota MAGs, which suggest that CR_14 belongs to a novel Bathyarchaeota

272 class (Table S2).

Metabolic analysis revealed that CR_14 harbors genes encoding for incomplete

274 methylotrophic methanogenesis pathway, yet is missing the key genes encoding for MCR

275 complex (mcrABCDG). The absence of this complex suggests that CR_14 is incapable of

276 methanogenesis and methane metabolic genes may be rewired to perform different functions, 
277 where they recycle methyl groups from different methylated compounds and replace the

278 functions of methyl branch in Wood-Ljungdahl pathway (Figure 4-A). CR_14 showed the

279 potential capability to use formate as electron and hydrogen donor through using Group 4

280 hydrogenases (formate hydrogenlyases) (Figure S6)[31], and these electrons reduce

281 trimethylamine N-oxide (TMAO) to trimethylamine via trimethylamine-oxide reductase or

282 anaerobic dimethyl sulfoxide reductase (TMAO/DMSO reductase)[34]. Together the presence of

283 genes encoding for trimethylamine-specific corrinoid protein as well as diverse classes of

284 methyltransferases, $\mathrm{CoB}$ - CoM heterodisulfide reductase/ F420 nonreducing hydrogenase

285 (hdrABCD and mvhADG) suggest the capability of CR_14 to recycle coenzyme M (CoM) and

286 coenzyme B (CoB) and transfer the methyl group from trimethylamine to CoM-SH[20][35]. We

287 located genes encoding the tetrahydromethanopterin S-methyltransferase (mtrA-H), suggesting

288 that mtrA protein transfers the methyl group from CoM to 5,6,7,8-Tetrahydromethanopterin

$289\left(\mathrm{H}_{4} \mathrm{MPT}\right)$ and assimilates the methyl group into acetyl-CoA via the beta subunit of CODH/ACS

290 complex, replacing the function of the methyl branch of Wood-Ljungdahl pathway. This agrees

291 with our finding that only the genes encoding for the carbonyl branch of Wood-Ljundahl coupled

292 with acetate fermentation genes (acetogenesis) were present in CR_14 and the genes encoding

293 for methyl branch were completely missing for the same pathway. These collective metabolic

294 features in Bathyarchaeota CR_14 suggest this genome may be a Bathyarchaeota lineage that

295 bridges the gap between methanogenic and acetogenic Bathyarchaeota through adopting a non-

296 canonical acetogenic life style (Table S5) [20][21].

Although the proposed scenario where CR_14 performs acetogenesis instead of

298 methanogenesis is metabolically feasible, it is not clear why CR_14 invests a large amount of

299 energy to maintain genes for methane metabolism. A plausible explanation for expressing 
300 methanogenesis-related genes is the lack of dedicated methyltransferases that could transport

301 methyl groups directly between methylated compounds (e.g. trimethylamine) to

302 Tetrahydromethanopterin (H4MPT). Therefore, encoding genes mediating the synthesis and

303 cycling of CoM is necessary to use it as intermediate carrier to transport methyl groups from/to

304 H4MPT. Also, the phylogenetic tree of the acetyl-CoA synthase beta subunit (AcsB) showed that

305 CR_14 AcsB genes clustered together with genes recovered from other Bathyarchaeota lineages,

306 Asgards, Chloroflexi and Altiarchaeales (Fig 4-B). The uniqueness of that clade stemmed from

307 the previous hypothesis that Altiarchaeales members possess an ancient version of AcsB [36].

308 Likewise, members of Chloroflexi encoded for this archaeal version of ACS $\beta$ subunit similar to

309 the ones present in Bathyarchaeota, Asgards, and Altiarchaeales [37][38]. Finally, this ACS type

310 supports the hydrogen dependent, autotrophic life style of Asgards [16]. Accordingly, we

311 hypothesize that Bathyarchaeota possess the type of ACS capable of incorporating methyl

312 groups into acetyl-CoA utilizing different carrier proteins (CoM, $\mathrm{H}_{4} \mathrm{MPT}$ and may be other

313 unknown carriers), allowing Bathyarchaeota to assimilate methyl groups originated from various

314 methylated compounds. 


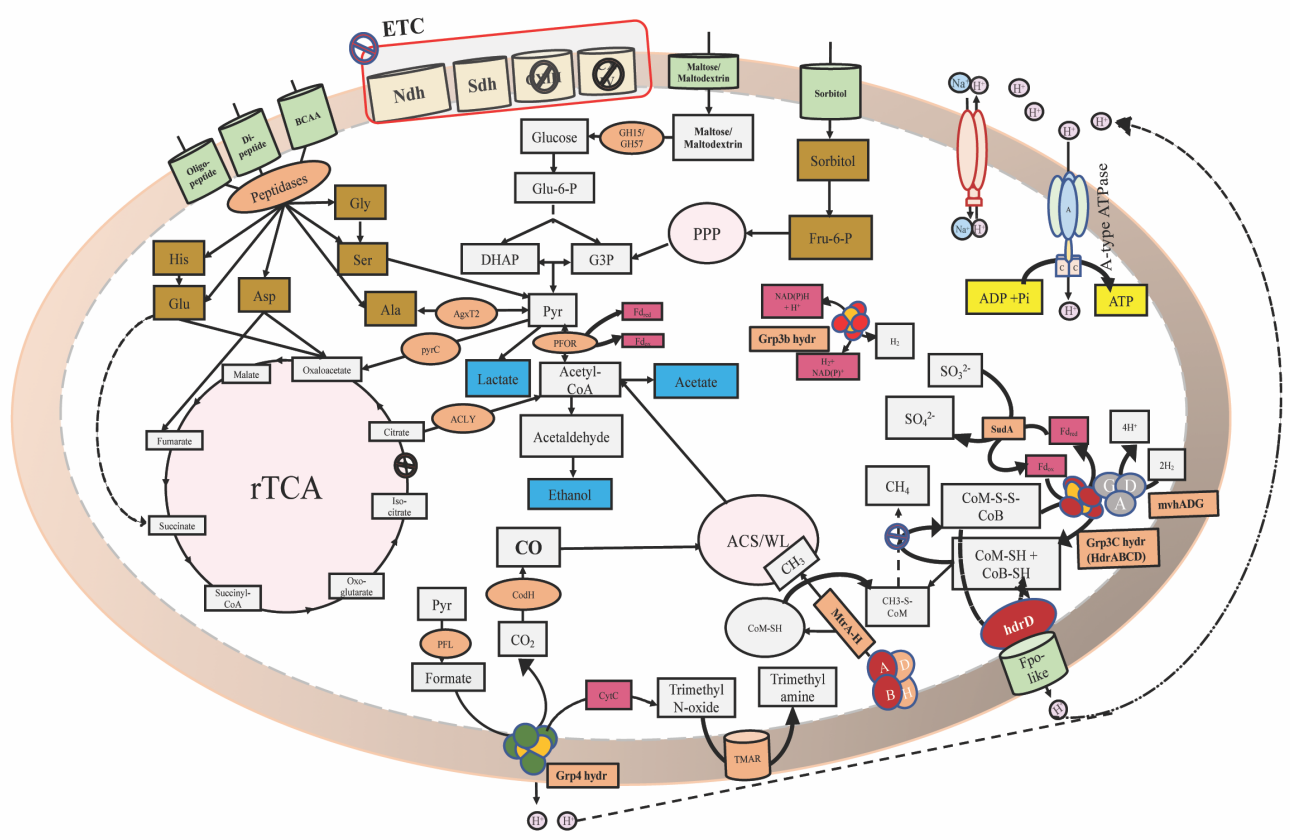

(B)

Tree scale: $0.1 \mapsto$

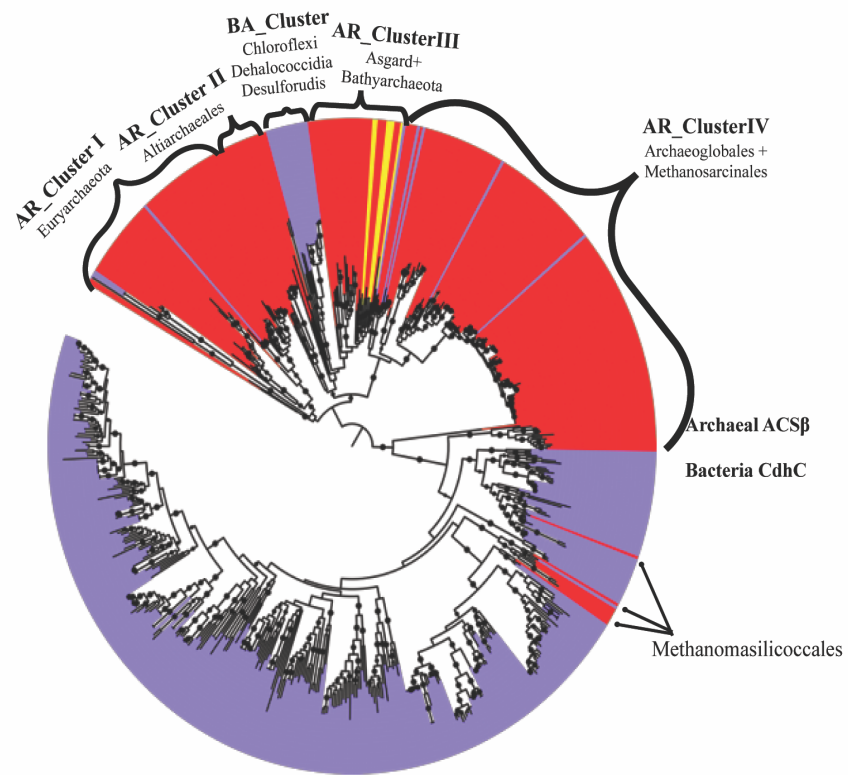

316 Figure 4. (A) Metabolic reconstruction of the Bathyarchaeota bin CR_14. Central metabolic pathways found in

317 the genome (glycolysis, carbonyl branch of Wood-Ljundahl, and methanogenesis related genes) are shown in gray

318 boxes, carbon fixation pathways (ACS/WL, PPP and rTCA cycles) are shown in pink, electron transport chain 
(ETC) proteins are shown in yellow, fermentation products are shown in blue boxes, amino acids are shown in brown boxes, enzymes and enzyme complexes are shown in orange circles, energy carriers are shown in red, metabolite and amino acid transporters are shown in light green. (B) Maximum likelihood tree of the acetyl CoA synthase $\boldsymbol{\beta}$ subunit $(\mathbf{A C S} \boldsymbol{\beta} / \mathbf{C d h C})$. The tree was calculated using the best fit substitution model $(\mathrm{LG}+\mathrm{R} 9)$ that describes the evolutionary relationships between ACS families. The tree was made using reference sequences under the KEGG entry (K00193) collected from AnnoTree[30] and branch location was tested using 1000 ultrafast bootstraps and approximate Bayesian computation, branches with bootstrap support $>80 \%$ were marked by black circles. Blue and Red clades highlight sequences belong to bacterial (CdhC) and archaeal (ACS $\beta$ ) versions,

327 respectively. Scale bar indicates substitutions per site. Sequences from CR_Lokiarchaeota and CR_Bathyarchaeota 328 bins were shaded with yellow. The tree is available with full bootstrap values in Newick format in the 329 Supplementary Data.

\section{Discussion}

In this study, we employed a metagenomics-enabled genomics approach to recover representative MAGs from the abundant archaeal phyla inhabiting deep sediments of Costa Rica margin and elucidate their potential ecological roles. A total of $31 \mathrm{MAGs}$ belonging to archaeal phyla (Lokiarchaeota, Thorarchaeota, Heimdallarchaeota, Bathyarcheota, Thermoplasmatales and Hadesarchaea) were successfully recovered from five metagenomic datasets representing

337 five different samples. Only 11 MAGs met our completion and contamination thresholds, $>60 \%$

338 complete and $<10 \%$ contamination, to be considered for the detailed genomic analyses and

339 metabolic reconstruction. More than $90 \%$ of the high-quality genomes were affiliated to

340 Bathyarchaeota and Asgard and the phylogenetic affiliations of the predicted proteins in each

341 MAG confirmed the proper quality of the MAGs considered in this study. Remarkably, all the

342 CR archaeal MAGs were enriched with ESP encoding genes. The wide distribution of these 
343 eukaryotic homologs indicates that ESPs are more ubiquitous in anaerobic archaea than

344 previously recognized.

Notably, the sediments used in this study were collected from much deeper sites compared to the sediment where previously reported Bathyarchaeota and Lokiarchaeota were

347 found. As such, our analysis was focused on MAGs belonging to Bathyarchaeota and

348 Lokiarchaeota to try to understand their ecological potentials under these deep marine sediment 349 conditions.

350 Community interactions and Lokiarchaeota metabolic interdependencies associated microbes with a significant portion of their energy and carbon needs [39][40].

353 Previous studies showed that hydrocarbon degradation is restricted to limited bacterial and 354 archaeal phyla (e.g. Aminicenantes, TA06, Aerophobetes, Atribacteria, Helarchaeota and 355 Bathyarchaeota) [18][20] [41]. However, the full spectrum of archaea involved in the 356 hydrocarbon degradation processes and the nature of their interactions are not fully elucidated. In 357 the light of the above evidence, this study provides new views regarding the ecological roles and 358 potential metabolic capacities of Lokiarcaheota. CR_Lokiarchaeota genome analysis expanded

359 the range of metabolic features encoded by this phylum and predicts metabolic functions 360 enabling the utilization of aliphatic and aromatic hydrocarbons as carbon and energy sources.

361 Considering the high energy demands for aromatic hydrocarbon breakdown under the energy

362 limited conditions of subseafloor sediments [28], it was unexpected to find the ATP-dependent

363 BCR complex (class Bzd) in CR-Asgards/CR-Lokiarchaeota genomes while they have

364 fermentation and/or acetogenic life styles. This strongly suggests that acetogenesis or acetate

365 fermentation cannot be the ultimate fate of the aromatic hydrocarbon degradation process due to 
366 the low energy yields of these pathways. Under these circumstances, we propose that the CR-

367 Lokiarchaeota members have the capacity of completely mineralizing aromatic hydrocarbons in

368 syntrophy with microbes capable of using nitrite, sulfite and nitrate as electron sink under the

369 subsurface settings to increase their energy budgets and sustain their energy requirement. After

370 testing all possible partnership scenarios based on the presence/absence profiles of the candidate

371 pathways and the thermodynamic feasibility (Figure 5 A-B), we found many potential partners

372 for Lokiarchaeota belong to diverse metabolic groups (e.g. nitrate reducers, nitrite reducers,

373 sulfate reducers, sulfite reducers and thiosulfate reducers), however only sulfite, nitrate and

374 nitrite reducers are thermodynamically favored. In the same context, a diverse group of

375 syntrophs might require sharing substrates of different qualities (e.g. acetate, propanoate, and

376 other short chain fatty acids). Accordingly, this suggests the presence of Lokiarchaeota

377 syntrophic partners capable of acetate oxidation and short chain fatty acid oxidation in addition

378 to the hydrogenotrophic ones as previously proposed [16]. Therefore, we hypothesize that

379 thermodynamic favorability and potential diversity of the shared metabolites will push the

380 Lokiarchaeota to syntrophy, beyond individual metabolite exchange.

\section{CR-Bathyarchaeota bridge the gap between acetogenic and methanogenic lineages}

382 Bathyarchaeota have been shown to have both methanogenic and acetogenic lifestyles [20][21],

383 yet the evolutionary trajectory and the ecological context driving the switch between lifestyles

384 are not fully understood. Generally, Bathyarchaeota lineages are widely distributed in different

385 benthic marine habitats compared to methanogenic ones [19], which could be explained by their

386 acetogenesis capacity that enables the degradation of wide range of organic compounds under

387 thermodynamic favorable conditions [42]. 
Interestingly, phylogenomic analysis revealed that CR_Bathyarchaeota MAGs are

389 potentially affiliated to novel class that are significantly divergent from previously reported

390 acetogenic and methanogenic lineages. In these CR sediments, distinct environmental conditions

391 may have captured the intermediate transition between acetogenic and methanogenic lifestyles.

392 We propose that these environmental conditions include the presence of high levels of

393 methylated compounds (e.g. methylated amines) in deep-sea environments [43] and the absence

394 of dedicated pathways/carriers necessary to recycle these methylated compounds and directly

395 shuttle the extracted methyl groups to the Wood-Ljungdahl pathway. Also, there is a relatively

396 high redox potential and substantial abundance of oxidized substrates that could serve as

397 terminal electron acceptors in CR sediments, this may not favor the occurrence of full

398 methanogenesis and the associated methanogenic archaea, as indicated by the absence of $m c r A$

399 genes at the sampled depths (Figure 5A).

400 In summary, this study presents a large dataset of subsurface archaeal MAGs, some of

401 which are high completeness and quality. Numerous archaeal MAGs host eukaryotic signatures,

402 yet, only the Asgard genomes have the homologs for cell division and cytoskeleton. We also

403 report a metabolically novel Lokiarchaeota lineage capable of aliphatic and aromatic

404 hydrocarbon degradation with a putative partnership with metabolically diverse syntrophic

405 organisms. Also, we revealed the presence of intermediate stage between acetogenic and

406 methanogenic Bathyarchaeota that could convert methylated amines to acetate through linking

407 methylotrophy to acetogenesis. 
bioRxiv preprint doi: https://doi.org/10.1101/763623; this version posted September 9, 2019. The copyright holder for this preprint (which was not certified by peer review) is the author/funder, who has granted bioRxiv a license to display the preprint in perpetuity. It is made available under aCC-BY-NC-ND 4.0 International license.

(A)
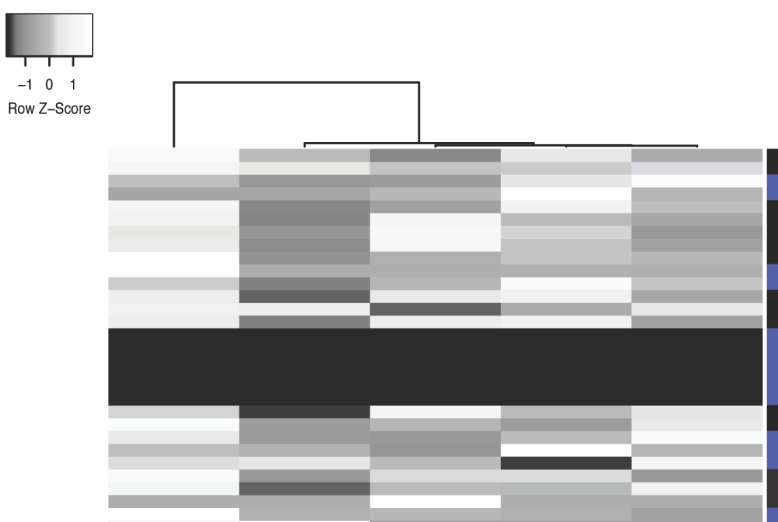
TCA

WL

Aromatic hydrocarbon degradation

Aliphatic hydrocarbon degradation $\beta$-oxidation

Methane metabolism

Sugar and protein degradation

Assimilatory sulfate reduction

Dissimilatory sulfate/sulfite reduction

Thiolsulfate reduction

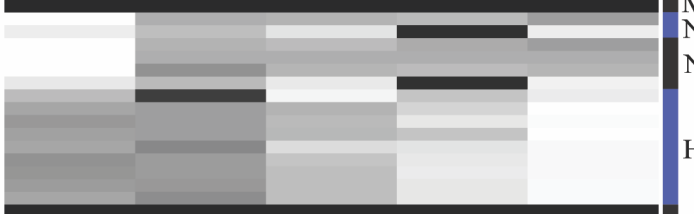

(n)

Nitrite reduction

Nitrate reduction

Hydrogen metabolims

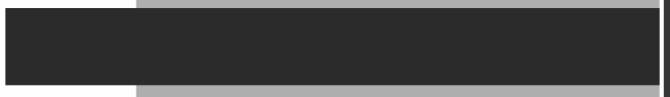

Oxygen metabolism

\begin{tabular}{|c|c|c|c|c|}
\hline $\begin{array}{l}w \\
w \\
\tilde{n} \\
\sim \\
\infty \\
\infty \\
\infty\end{array}$ & 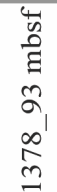 & 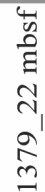 & $\begin{array}{l}4 \\
\text { n } \\
\text { है } \\
m \\
m \\
\infty \\
\infty \\
m\end{array}$ & $\begin{array}{l}\text { w } \\
\text { है } \\
\text { n }\end{array}$ \\
\hline
\end{tabular}

(B)

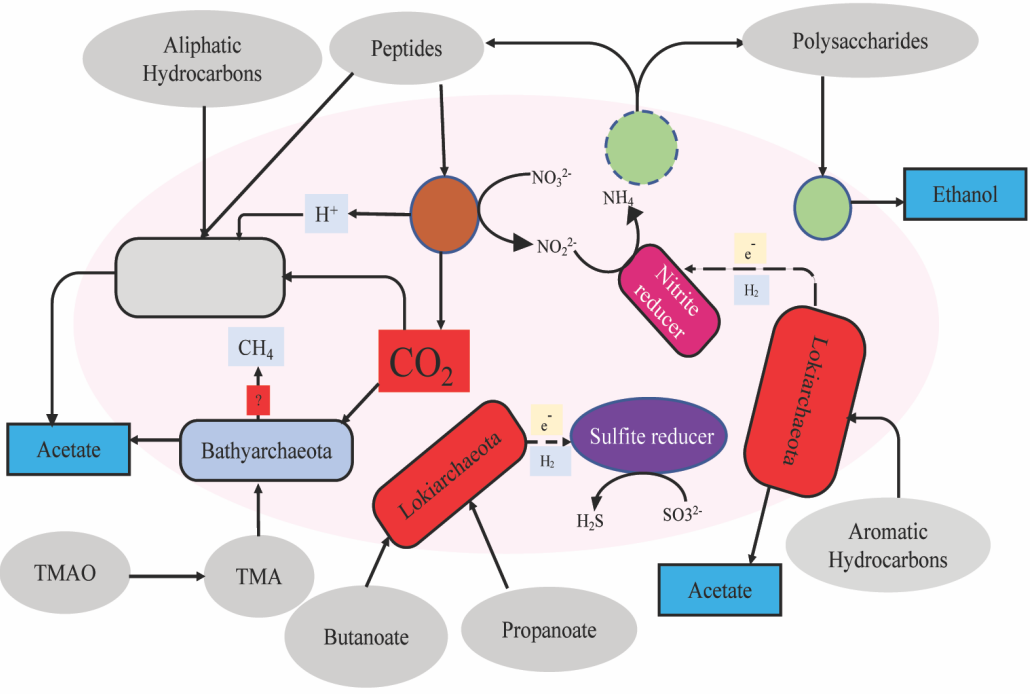


409 Figure 5. (A) Heat map depicts the distribution patterns of the key metabolic pathways across all sampled

410 metagenomic datasets. Metagenomes were clustered based on presence (black) /absence (white) profiles using

411 Euclidean distance and average linkage method. Y axis represents pathways included in the analysis and $\mathrm{x}$ axis

412 includes metagenomic datasets analyzed. (B) Hypothetical models for the microbial interactions in the Costa

413 Rica sediments. The potential substrates degraded by Lokiarchaeota, Bathyarchaeota and other microbes in the

414 environment were colored in grey, potential metabolic products (in blue), potential interactions between

415 Lokiarchaeota and their syntrophic partners and possible shared metabolites 


\section{Materials and Methods}

\section{Site information and Sample collection}

Samples used in this study were collected under aseptic conditions from Sites U1378 and

419 U1379 of Costa Rica Margin during IODP Expedition 334. The sample depths are in the range of

$4202-93$ meters below seafloor (mbsf). Detailed site descriptions were previously reported in the

421 IODP Proceedings for Expedition 334 [44][22].

\section{DNA Extraction and Sequencing and genomes binning}

DNA extraction and metagenomic sequencing have been described previously [22] and

424 data have been deposited at NCBI GenBank SRA under project PRJEB11766. Metagenomic

425 reads were quality trimmed using Nesoni following default parameter and applying q20 for

426 quality score (www.vicbioinformatics.com/software.nesoni.shtml). Quality-controlled reads in

427 individual samples were assembled separately using IDBA-UD[45] with default settings. Contigs

428 longer than $1 \mathrm{~kb}$ were binned into MAGs using MaxBin V2.2.7[46] and further curated manually

429 using VizBin[47] and through filtering outlier scaffolds not falling within the same GC\% and

430 differential coverage levels across different Costa Rica datasets. The quality and completeness of

431 the MAGs were assessed using CheckM (v.1.0.7) [48]. MAGs from all give samples were de-

432 replicated using dRep (version v2.0.5 with ANI > 99\%)[49] and most complete MAG per taxon

433 was selected for downstream analyses. Archaeal bins were further analyzed to determine their

434 phylogenetic placements through the analysis of single copy marker genes using Phylosift[50]

435 and 16 ribosomal proteins (see description below). Assembled contigs larger than $1 \mathrm{~kb}$ were

436 annotated using PROKKA[51]. Encoded proteins were predicted using Prodigal v2.6.3 with the

437 default translation table (table 11) was applied[52].

\section{Concatenated ribosomal protein phylogeny}


439 A maximum-likelihood tree was calculated based on the concatenation of 16 ribosomal proteins

440 (L2, L3, L4, L5, L6, L14, L15, L16, L18, L22, L24, S3, S8, S10, S17, and S19) using IQ-

441 Tree[53] (located on the CIPRES web server)[54]. References sequences used were collected

442 [12] with the addition of more representatives of the Bathyarchaeota and Asgard lineages.

443 Evolutionary distances were calculated based on best fit substitution model (VT+F+R10), and

444 single branch location was tested using 1000 ultrafast bootstraps and approximate Bayesian

445 computation[55][53], branches with bootstrap support $>80 \%$ were marked by black circles.

446 Metabolic reconstruction and Functional annotation

447 Predicted proteins from all MAGs were screened using HMMsearch tool against custom

448 HMM databases representing the key genes for specific metabolic pathways [56]. The

449 completion of the pathways was assessed through querying the predicted proteins against KEGG

450 database using BlastKoala tool[57]. Carbohydrate-active enzymes (CAZymes) were identified

451 using dbCAN-fam-HMMs (v6) database[58]. Cellular localizations of predicted proteins were

452 identified using SignalP v5.0[59]. Proteases, peptidases, and peptidase inhibitors were identified

453 using USEARCH-ublast tool[60] against the MEROPS database v12.1[61]. Transporters were

454 identified using USEARCH-ublast tool[60] against the TCDB database[62]. Eukaryotic signature

455 proteins were detected using Interpro v75.0[63]. Phylogenetic distributions of the predicted

456 proteins in each bin were detected through comparing these proteins against the NCBI (nr)

457 protein database via the DarkHorse software[23].

$458 \quad$ Functional proteins-based trees

459 All functional proteins-based trees were built by aligning the query proteins sequences to the

460 reference sequences belonging to the same protein family using Muscle v3.8.31[64]. Reference

461 sequences were collected from AnnoTree[30] using the corresponding KEGG entry as search 
462 keyword. Aligned sequences were manually curated using Geneious v9.0.5

463 (https://www.geneious.com). The phylogenetic trees were computed using IQ-TREE (v1.6.6)

464 [53], through the CIPRES web server[52] and the evolutionary relationships were described

465 using the best fit model. Branch locations were tested using 1000 ultrafast bootstraps and

466 approximate Bayesian computation[55][53].

\section{Thermodynamic calculations}

468 Initial Gibbs free energy $\left(\Delta \mathrm{rG}^{\prime}\right)$ calculations were performed for the redox reactions proposed in 469 the CR Lokiarchaeota and their poteintial partners using eQuilibrator[65] applying pH 8 similar 470 to the approach reported in[42] and reactant concentrations $1 \mathrm{mM}$ each.

471 We further confirmed if the coupled redox reactions proposed for the novel CR Lokiarchaeota

472 genomes were feasible, we calculated the Gibbs free energy of five redox reactions (Table S1)

473 under the near in situ conditions at CR core U1378 and U1379, following the method described

474 in LaRowe and Amend (2015)[66]. Gibbs free energy was calculated using the equation:

$475 \Delta G_{r}=\Delta G_{r}{ }^{0}+R T \operatorname{In} Q_{r}$

476 where $\Delta G_{r}{ }^{0}$ and $Q_{r}$ refer to the standard molar Gibbs energy and the reaction quotient of the

477 indicated reaction, respectively, $R$ represents the gas constant, and $T$ denotes temperature in

478 Kelvin. In this study, $\Delta G_{r}{ }^{0}$ was calculated using the thermodynamic data of standard Gibbs free

479 energy of formation of each species and corrected to near in situ pressure and temperature $\left(4^{\circ} \mathrm{C}\right)$,

480 using the $R$ package CHNOSZ [67]. Qr stands for the reaction quotient, which can be calculated

481 with the relation

$482 Q_{r}=\Pi\left(\mathrm{a}_{i}{ }^{v i}\right)$

483 where $\mathrm{a}_{i}$ is the activity of species $i$ and $v_{i}$ is its stoichiometric coefficient. $\mathrm{a}_{i}$ is the product of 484 chemical species concentration $[i]$ and its activity coefficient $\gamma_{i}$, which was computed as a function 485 of temperature and ionic strength by using an extended version of the Debye-Huckel equation[68]. 
486 Because most of the reactant concentrations were hard to measure or were below detection limits,

487 we assumed $0.1 \mu \mathrm{M}$ for the concentration of $\mathrm{NO}_{3}{ }^{-}, \mathrm{NO}_{2}{ }^{-}, \mathrm{SO}_{3}{ }^{2-}$, and $\mathrm{H}_{2} \mathrm{~S}$. We considered a wide

488 range of concentrations for benzoate $\left(\mathrm{C}_{7} \mathrm{H}_{5} \mathrm{O}_{2}{ }^{-}\right)(0.0001-100 \mu \mathrm{M})$, to explore the feasibility of

489 these reactions over a wide range of substrate concentration changes. 


\section{Acknowledgements}

492 This work was funded by IODP 334 Post Expedition award T334A40, Exxon Mobil Research

493 and Engineering, Penn State Astrobiology Research Center (through the NASA Astrobiology

494 Institute, cooperative agreement \#NNA09DA76A), the Pennsylvania Space Grant Consortium

495 (NNX10AK74H), and a postdoctoral fellowship (RLZ) and graduate student fellowship (AJM)

496 from NSF-funded Center for Dark Energy Biosphere investigations. This is CDEBI contribution

$497 \quad$ \# XXX.

498 We thank Frances O. Mark for valuable technical assistance.

499 Author contributions

500 J.B, I.F and R.L.Z. conceived the study. R.L.Z recovered the genomes from the metagenomic

501 datasets. I.F, R.L.Z analyzed the genomic data. A.M and C.H collected the samples. R.Z revised

502 the thermodynamic calculations and I.F., J.B and R.L.Z. wrote the manuscript with input from all

503 authors. All documents were edited and approved by all authors.

\section{Competing interests}

505 The other authors declare no competing interests.

\section{Materials and Correspondence}

507 Correspondence and material requests should be addressed to Jennifer Biddle

$508<$ jfbiddle@udel.edu>.

\section{Data availability}

510 The genomes of this study have been made publicly available on GenBank under BioSample

511 accessions (SAMN12695919- SAMN12695949). Metagenomes can be located at NCBI GenBank

512 SRA under project PRJEB11766. 


\section{References}

515 [1] A. Teske and K. B. Sørensen, "Uncultured archaea in deep marine subsurface sediments:

516 have we caught them all?," ISME J., vol. 2, no. 1, pp. 3-18, Jan. 2008.

517 [2] J. F. Biddle et al., "Heterotrophic Archaea dominate sedimentary subsurface ecosystems off

518 Peru," Proc. Natl. Acad. Sci. U. S. A., vol. 103, no. 10, pp. 3846-3851, Mar. 2006.

519 [3] A. Schippers et al., "Prokaryotic cells of the deep sub-seafloor biosphere identified as living bacteria," Nature, vol. 433, no. 7028, pp. 861-864, Feb. 2005.

521 [4] J. S. Lipp, Y. Morono, F. Inagaki, and K.-U. Hinrichs, "Significant contribution of Archaea to extant biomass in marine subsurface sediments," Nature, vol. 454, no. 7207, pp. 991-

524 [5] J. Buongiorno et al., "Interlaboratory quantification of Bacteria and Archaea in deeply buried sediments of the Baltic Sea (IODP Expedition 347)," FEMS Microbiol. Ecol., vol. 93, no. 3,

[6] J. F. Biddle, S. Fitz-Gibbon, S. C. Schuster, J. E. Brenchley, and C. H. House, "Metagenomic signatures of the Peru Margin subseafloor biosphere show a genetically distinct environment," Proc. Natl. Acad. Sci. U. S. A., vol. 105, no. 30, pp. 10583-10588, Jul. 2008.

[7] N. H. Youssef, C. Rinke, R. Stepanauskas, I. Farag, T. Woyke, and M. S. Elshahed, "Insights into the metabolism, lifestyle and putative evolutionary history of the novel archaeal

533 [8] I. F. Farag, N. H. Youssef, and M. S. Elshahed, “Global Distribution Patterns and Microbiol., vol. 83, no. 10, 152017. 
[9] K. Kubo, K. G. Lloyd, J. F Biddle, R. Amann, A. Teske, and K. Knittel, “Archaea of the Miscellaneous Crenarchaeotal Group are abundant, diverse and widespread in marine sediments," ISME J., vol. 6, no. 10, pp. 1949-1965, Oct. 2012.

[10] C. Rinke et al., "Insights into the phylogeny and coding potential of microbial dark matter," Nature, vol. 499, no. 7459, pp. 431-437, Jul. 2013.

[11] A. Spang et al., "Complex archaea that bridge the gap between prokaryotes and eukaryotes," Nature, vol. 521, no. 7551, pp. 173-179, May 2015.

[12] L. A. Hug et al., "A new view of the tree of life," Nat. Microbiol., vol. 1, p. 16048, Apr. 2016.

[13] C. J. Castelle and J. F. Banfield, "Major New Microbial Groups Expand Diversity and Alter our Understanding of the Tree of Life," Cell, vol. 172, no. 6, pp. 1181-1197, 082018.

[14] K. Anantharaman et al., "Thousands of microbial genomes shed light on interconnected biogeochemical processes in an aquifer system," Nat. Commun., vol. 7, p. 13219, 242016.

[15] K. Zaremba-Niedzwiedzka et al., "Asgard archaea illuminate the origin of eukaryotic cellular complexity," Nature, vol. 541, no. 7637, pp. 353-358, 192017.

[16] A. Spang et al., "Proposal of the reverse flow model for the origin of the eukaryotic cell based on comparative analyses of Asgard archaeal metabolism," Nat. Microbiol., vol. 4, no.

554 [17] F. MacLeod, G. S. Kindler, H. L. Wong, R. Chen, and B. P. Burns, “Asgard archaea: 555 Diversity, function, and evolutionary implications in a range of microbiomes," AIMS Microbiol., vol. 5, no. 1, pp. 48-61, 2019.

557 [18] K. W. Seitz et al., “Asgard archaea capable of anaerobic hydrocarbon cycling,” Nat.

558 Commun., vol. 10, no. 1, p. 1822, 232019. 
[19] Z. Zhou, J. Pan, F. Wang, J.-D. Gu, and M. Li, "Bathyarchaeota: globally distributed metabolic generalists in anoxic environments," FEMS Microbiol. Rev., vol. 42, no. 5, pp. 639-655, 012018.

[20] P. N. Evans et al., "Methane metabolism in the archaeal phylum Bathyarchaeota revealed by genome-centric metagenomics," Science, vol. 350, no. 6259, pp. 434-438, Oct. 2015.

[21] Y. He et al., "Genomic and enzymatic evidence for acetogenesis among multiple lineages of the archaeal phylum Bathyarchaeota widespread in marine sediments," Nat. Microbiol., vol. 1, no. 6, p. 16035, 042016.

[22] Amanda Martino, Matthew E. Rhodes, Rosa León-Zayas, Isabella E. Valente, Jennifer F. Biddle and Christopher H. House, "Microbial Diversity in Sub-Seafloor Sediments from the Costa Rica Margin,” Geosciences, vol. 9, p. 218, 2019.

[23] S. Podell and T. Gaasterland, "DarkHorse: a method for genome-wide prediction of

[24] L. A. Levin et al., "A hydrothermal seep on the Costa Rica margin: middle ground in a continuum of reducing ecosystems," Proc. Biol. Sci., vol. 279, no. 1738, pp. 2580-2588, Jul. 2012.

[25] R. U. Meckenstock et al., "Anaerobic Degradation of Benzene and Polycyclic Aromatic Hydrocarbons," J. Mol. Microbiol. Biotechnol., vol. 26, no. 1-3, pp. 92-118, 2016. 
[27] B. Song and B. B. Ward, "Genetic diversity of benzoyl coenzyme A reductase genes detected in denitrifying isolates and estuarine sediment communities," Appl. Environ. Microbiol., vol. 71, no. 4, pp. 2036-2045, Apr. 2005.

[28] M. J. López Barragán et al., "The bzd gene cluster, coding for anaerobic benzoate catabolism, in Azoarcus sp. strain CIB,” J. Bacteriol., vol. 186, no. 17, pp. 5762-5774, Sep. 2004.

[29] J.-M. Huang, B. J. Baker, J.-T. Li, and Y. Wang, "New Microbial Lineages Capable of Carbon Fixation and Nutrient Cycling in Deep-Sea Sediments of the Northern South China

[30] K. Mendler, H. Chen, D. H. Parks, B. Lobb, L. A. Hug, and A. C. Doxey, “AnnoTree: visualization and exploration of a functionally annotated microbial tree of life," Nucleic Acids Res., vol. 47, no. 9, pp. 4442-4448, May 2019.

[31] C. Greening et al., "Genomic and metagenomic surveys of hydrogenase distribution indicate $\mathrm{H} 2$ is a widely utilised energy source for microbial growth and survival," ISME J., vol. 10, no. 3, pp. 761-777, Mar. 2016.

[32] A. Kouzuma, S. Kato, and K. Watanabe, "Microbial interspecies interactions: recent

597 [33] S. Pirbadian et al., "Shewanella oneidensis MR-1 nanowires are outer membrane and 
602

603

604

605

606

607

608

609

610

[35] P. N. Evans et al., "An evolving view of methane metabolism in the Archaea," Nat. Rev. Microbiol., vol. 17, no. 4, pp. 219-232, Apr. 2019.

[36] P. S. Adam, G. Borrel, and S. Gribaldo, "Evolutionary history of carbon monoxide dehydrogenase/acetyl-CoA synthase, one of the oldest enzymatic complexes," Proc. Natl. Acad. Sci. U. S. A., vol. 115, no. 6, pp. E1166-E1173, 062018.

[37] L. A. Hug et al., "Community genomic analyses constrain the distribution of metabolic traits across the Chloroflexi phylum and indicate roles in sediment carbon cycling," Microbiome, vol. 1, no. 1, p. 22, Aug. 2013.

[38] M. Mehrshad et al., "Hidden in plain sight-highly abundant and diverse planktonic freshwater Chloroflexi," Microbiome, vol. 6, no. 1, p. 176, 022018.

[39] B. B. Jørgensen and A. Boetius, "Feast and famine--microbial life in the deep-sea bed," Nat. Rev. Microbiol., vol. 5, no. 10, pp. 770-781, Oct. 2007.

[40] X. Dong et al., "Metabolic potential of uncultured bacteria and archaea associated with petroleum seepage in deep-sea sediments," Nat. Commun., vol. 10, no. 1, p. 1816, 182019.

[41] Y.-F. Liu et al., "Anaerobic hydrocarbon degradation in candidate phylum 'Atribacteria' (JS1) inferred from genomics," ISME J., Jun. 2019.

[42] M. A. Lever, "Acetogenesis in the energy-starved deep biosphere - a paradox?," Front. Microbiol., vol. 2, p. 284, 2011.

[43] M. A. Mausz and Y. Chen, "Microbiology and Ecology of Methylated Amine Metabolism in Marine Ecosystems," Curr. Issues Mol. Biol., vol. 33, pp. 133-148, Jun. 2019.

[44] Vannucchi, P.; Ujiie, K.; Stroncik, N.; IODP Exp. 334 Scientific Party; Yatheesh, V., "IODP expedition 334: An investigation of the sedimentary record, fluid flow and state of 
stress on top of the seismogenic zone of an erosive subduction margin," Sci. Drill., vol. vol.15, pp. 23-30, 2013.

[45] Y. Peng, H. C. M. Leung, S. M. Yiu, and F. Y. L. Chin, "IDBA-UD: a de novo assembler for single-cell and metagenomic sequencing data with highly uneven depth," Bioinforma. Oxf. Engl., vol. 28, no. 11, pp. 1420-1428, Jun. 2012.

[46] Y.-W. Wu, Y.-H. Tang, S. G. Tringe, B. A. Simmons, and S. W. Singer, "MaxBin: an automated binning method to recover individual genomes from metagenomes using an expectation-maximization algorithm," Microbiome, vol. 2, p. 26, 2014.

[47] C. C. Laczny et al., "VizBin - an application for reference-independent visualization and human-augmented binning of metagenomic data," Microbiome, vol. 3, no. 1, p. 1, 2015.

[48] D. H. Parks, M. Imelfort, C. T. Skennerton, P. Hugenholtz, and G. W. Tyson, "CheckM: assessing the quality of microbial genomes recovered from isolates, single cells, and metagenomes," Genome Res., vol. 25, no. 7, pp. 1043-1055, Jul. 2015.

[49] M. R. Olm, C. T. Brown, B. Brooks, and J. F. Banfield, "dRep: a tool for fast and accurate genomic comparisons that enables improved genome recovery from metagenomes through de-replication," ISME J., vol. 11, no. 12, pp. 2864-2868, 2017.

[50] A. E. Darling, G. Jospin, E. Lowe, F. A. Matsen, H. M. Bik, and J. A. Eisen, "PhyloSift: phylogenetic analysis of genomes and metagenomes," PeerJ, vol. 2, p. e243, 2014.

[51] T. Seemann, "Prokka: rapid prokaryotic genome annotation," Bioinforma. Oxf. Engl., vol. 30, no. 14, pp. 2068-2069, Jul. 2014.

[52] D. Hyatt, G.-L. Chen, P. F. Locascio, M. L. Land, F. W. Larimer, and L. J. Hauser, "Prodigal: prokaryotic gene recognition and translation initiation site identification," BMC Bioinformatics, vol. 11, p. 119, Mar. 2010. 
[53] L.-T. Nguyen, H. A. Schmidt, A. von Haeseler, and B. Q. Minh, "IQ-TREE: a fast and effective stochastic algorithm for estimating maximum-likelihood phylogenies," Mol. Biol. Evol., vol. 32, no. 1, pp. 268-274, Jan. 2015.

[54] M. A. Miller, W. Pfeiffer, and T. Schwartz, "Creating the CIPRES Science Gateway for inference of large phylogenetic trees," 2010 Gatew. Comput. Environ. Workshop GCE, pp. $1-8,2010$.

[55] D. T. Hoang, O. Chernomor, A. von Haeseler, B. Q. Minh, and L. S. Vinh, "UFBoot2: Improving the Ultrafast Bootstrap Approximation,” Mol. Biol. Evol., vol. 35, no. 2, pp. 518-522, 012018.

[56] L. S. Johnson, S. R. Eddy, and E. Portugaly, "Hidden Markov model speed heuristic and iterative HMM search procedure," BMC Bioinformatics, vol. 11, p. 431, Aug. 2010.

[57] M. Kanehisa, Y. Sato, and K. Morishima, "BlastKOALA and GhostKOALA: KEGG Tools for Functional Characterization of Genome and Metagenome Sequences," J. Mol. Biol., vol. 428, no. 4, pp. 726-731, Feb. 2016.

[58] Y. Yin, X. Mao, J. Yang, X. Chen, F. Mao, and Y. Xu, "dbCAN: a web resource for automated carbohydrate-active enzyme annotation," Nucleic Acids Res., vol. 40, no. Web Server issue, pp. W445-451, Jul. 2012.

[59] J. J. Almagro Armenteros et al., "SignalP 5.0 improves signal peptide predictions using deep neural networks," Nat. Biotechnol., vol. 37, no. 4, pp. 420-423, 2019.

[60] R. C. Edgar, "Search and clustering orders of magnitude faster than BLAST," Bioinforma. Oxf. Engl., vol. 26, no. 19, pp. 2460-2461, Oct. 2010.

[61] N. D. Rawlings, A. J. Barrett, P. D. Thomas, X. Huang, A. Bateman, and R. D. Finn, "The MEROPS database of proteolytic enzymes, their substrates and inhibitors in 2017 and a 
comparison with peptidases in the PANTHER database," Nucleic Acids Res., vol. 46, no. D1, pp. D624-D632, 042018.

672 [62] M. H. Saier, V. S. Reddy, B. V. Tsu, M. S. Ahmed, C. Li, and G. Moreno-Hagelsieb, “The

673 Transporter Classification Database (TCDB): recent advances," Nucleic Acids Res., vol. 44, 674 no. D1, pp. D372-379, Jan. 2016.

675 [63] P. Jones et al., "InterProScan 5: genome-scale protein function classification,” Bioinforma. Oxf. Engl., vol. 30, no. 9, pp. 1236-1240, May 2014.

677 [64] R. C. Edgar, "MUSCLE: multiple sequence alignment with high accuracy and high throughput," Nucleic Acids Res., vol. 32, no. 5, pp. 1792-1797, 2004.

[65] A. Flamholz, E. Noor, A. Bar-Even, and R. Milo, "eQuilibrator--the biochemical thermodynamics calculator," Nucleic Acids Res., vol. 40, no. Database issue, pp. D770-775,

[66] D. E. LaRowe and J. P. Amend, "Catabolic rates, population sizes and doubling/replacement times of microorganisms in natural settings,” Am. J. Sci., vol. 315, pp. 167-203, Mar. 2015.

[67] J. M. Dick, "Calculation of the relative metastabilities of proteins using the CHNOSZ software package," Geochem. Trans., vol. 9, p. 10, Oct. 2008.

687 [68] H. C. Helgeson, "Thermodynamics of hydrothermal systems at elevated temperatures and pressures,” Am. J. Sci., vol. 267, pp. 729-804, Sep. 1969. 
Table 1. Details of the genomes constructed from the Costa Rica Margin

\begin{tabular}{|c|c|c|c|c|c|c|c|c|c|c|c|c|}
\hline Bin_ID & Dataset & Marker lineage & Phylogenetic affiliation & Completeness & completeness_metric & Contamination & $\begin{array}{c}\text { Strain } \\
\text { heterogeneity }\end{array}$ & $\begin{array}{l}\text { Genome } \\
\text { size } \\
(\mathrm{Mbp})\end{array}$ & $\begin{array}{c}\# \\
\text { scaffolds }\end{array}$ & GC & $\begin{array}{c}\text { GC std } \\
\text { (scaffolds } \\
>1 \mathrm{kbp})\end{array}$ & Coverage \\
\hline CR_01 & 1378_2mbsf & k__Archaea (UID2) & Bathyarchaeota & 38.71 & partial & 10.09 & 59.09 & 0.62 & 335 & 39 & 6.02 & 155.6 \\
\hline CR_02 & $1378 \_2 \mathrm{mbsf}$ & $\mathrm{k} \quad$ Archaea (UID2) & Bathyarchaeota & 45.64 & partial & 5.61 & 16.67 & 1.05 & 225 & 38.7 & 0 & 29.9 \\
\hline CR_03 & 1378_2mbsf & $\begin{array}{l}\mathrm{p} \_ \text {Euryarchaeota } \\
\text { (UID3) }\end{array}$ & Hadesarchaea & 74.86 & substantial & 10.67 & 50 & 0.98 & 333 & 40.2 & 6.68 & 19.3 \\
\hline CR_04 & 1378_2mbsf & $\mathrm{k} \quad$ Archaea (UID2) & Bathyarchaeota & 60.62 & moderate & 10.08 & 5.88 & 1.09 & 368 & 42.7 & 4.79 & 16.05 \\
\hline CR_05 & 1378_2mbsf & $\mathrm{k} \quad$ Archaea (UID2) & Bathyarchaeota (RPL2) & 65.2 & moderate & 10.26 & 0 & 1.92 & 900 & 40.9 & 0 & 11.4 \\
\hline CR_06 & $1378 \_32 \mathrm{mbsf}$ & $\mathrm{k} \quad$ Archaea (UID2) & Asgard (Lokiarchaeota) & 86.78 & substantial & 1.87 & 33.33 & 2.41 & 1110 & 42.7 & 11.06 & 216.64 \\
\hline CR_07 & $1378 \_32 \mathrm{mbsf}$ & $\mathrm{k} \quad$ Archaea (UID2) & Asgard (Lokiarchaeota) & 47.66 & partial & 5.43 & 63.64 & 1.525 & 829 & 41.9 & 0 & 72.54 \\
\hline CR_08 & $1378 \_32 \mathrm{mbsf}$ & k_ Archaea (UID2) & Asgard (Lokiarchaeota) & 61.06 & moderate & 9.65 & 20 & 3.54 & 693 & 42.9 & 8.76 & 71.06 \\
\hline CR_09 & 1378_32mbsf & $\mathrm{k} \quad$ Archaea (UID2) & Bathyarchaeota & 41.64 & partial & 0 & 0 & 3.6 & 89 & 39.3 & 0 & 30.63 \\
\hline CR_10 & 1378_32mbsf & $\mathrm{k}$ _Archaea (UID2) & Asgard (Lokiarchaeota) & 32.81 & partial & 10.6 & 23.33 & 2.91 & 1865 & 43.3 & 6.55 & 24.53 \\
\hline CR_11 & $1378 \_32 \mathrm{mbsf}$ & $\mathrm{k} \quad$ Archaea (UID2) & $\begin{array}{c}\text { Asgard } \\
\text { (Heimdallarchaeota) }\end{array}$ & 94.39 & near & 9.06 & 0 & 3.47 & 167 & 38.6 & 7.88 & 23.11 \\
\hline CR_12 & $1378 \_32 \mathrm{mbsf}$ & $\mathrm{k} \quad$ Archaea (UID2) & Asgard (Thorarchaeota) & 32.05 & partial & 1.08 & 0 & 1.49 & 1019 & 38.8 & 0 & 10.48 \\
\hline CR_13 & 1379_22mbsf & $\mathrm{k} \quad$ Archaea (UID2) & Bathyarchaeota & 35.05 & partial & 0 & 0 & 0.3 & 118 & 39.2 & 0 & 116.39 \\
\hline CR_14 & 1379_22mbsf & $\mathrm{k} \quad$ Archaea (UID2) & Bathyarchaeota & 95.64 & near & 4.15 & 0 & 2.49 & 140 & 39.4 & 7.77 & 77.12 \\
\hline CR_15 & $1379 \_22 \mathrm{mbsf}$ & $\mathrm{k} \quad$ Archaea (UID2) & Bathyarchaeota & 92.61 & near & 3.12 & 0 & 2.11 & 130 & 39 & 5.31 & 47.44 \\
\hline CR_16 & 1379_22mbsf & $\mathrm{k}$ Archaea (UID2) & Bathyarchaeota & 36.14 & partial & 0 & 0 & 2.99 & 59 & 39.4 & 7.03 & 47.78 \\
\hline CR_17 & 1379_22mbsf & k__Archaea (UID2) & Bathyarchaeota & 71.03 & substantial & 6.54 & 0 & 0.88 & 28 & 39.4 & 7.51 & 26.16 \\
\hline CR_18 & $1379 \_22 \mathrm{mbsf}$ & $\mathrm{k} \quad$ Archaea (UID2) & Bathyarchaeota (RPL2) & 42.3 & partial & 5.61 & 0 & 1.13 & 103 & 40.8 & 6.46 & 24.29 \\
\hline CR_19 & 1379_22mbsf & k_Archaea (UID2) & Bathyarchaeota & 35.84 & partial & 5.99 & 0 & 1.03 & 340 & 41 & 0 & 13.83 \\
\hline CR_20 & $1379 \_22 \mathrm{mbsf}$ & $\mathrm{k} \quad$ Archaea (UID2) & Bathyarchaeota & 58.35 & moderate & 2.8 & 0 & 1.01 & 184 & 41 & 6.38 & 18.65 \\
\hline CR_21 & 1379_22mbsf & $\mathrm{k} \_$Archaea (UID2) & Bathyarchaeota & 38.51 & partial & 1.94 & 0 & 0.58 & 249 & 41.8 & 5.06 & 17.31 \\
\hline CR_22 & 1379_22mbsf & $\mathrm{k} \quad$ Archaea (UID2) & Bathyarchaeota & 42.88 & partial & 9.88 & 15.38 & 1.31 & 344 & 38.6 & 3.12 & 19.48 \\
\hline
\end{tabular}




\begin{tabular}{|c|c|c|c|c|c|c|c|c|c|c|c|c|}
\hline CR_23 & 1379_22mbsf & 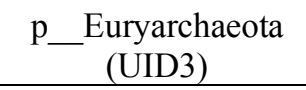 & Thermoplasmata & 62.44 & moderate & 2.4 & 0 & 1.96 & 284 & 43.1 & 5.55 & 19.88 \\
\hline CR_24 & 1379_22mbsf & $\mathrm{k}$ Archaea (UID2) & Bathyarchaeota & 53.74 & partial & 6.31 & 0 & 2.36 & 556 & 41.8 & 3.38 & 17.98 \\
\hline CR_25 & 1379_22mbsf & $\mathrm{k}$ Archaea (UID2) & Bathyarchaeota (RPS3) & 56.65 & moderate & 1.94 & 0 & 1.68 & 531 & 39.6 & 3.35 & 13.92 \\
\hline CR_26 & 1379_22mbsf & $\mathrm{k}$ Archaea (UID2) & Hadesarchaea & 50.96 & moderate & 7.79 & 0 & 0.58 & 329 & 39.6 & 0 & 14.9 \\
\hline CR_27 & $1379 \_45 \mathrm{mbsf}$ & $\mathrm{k}$ Archaea (UID2) & Bathyarchaeota & 79.77 & substantial & 5.49 & 33.33 & 1.24 & 404 & 39.8 & 4.19 & 63.85 \\
\hline CR_28 & $1379 \_45 \mathrm{mbsf}$ & $\mathrm{k}$ Archaea (UID2) & Bathyarchaeota & 32.78 & partial & 6.15 & 0 & 1.25 & 296 & 39.1 & 8.11 & 45.38 \\
\hline CR_29 & $1379 \_45 \mathrm{mbsf}$ & $\mathrm{k} \quad$ Archaea (UID2) & Bathyarchaeota & 56.96 & moderate & 0.97 & 100 & 0.7 & 154 & 37.7 & 7.52 & 40.92 \\
\hline CR_30 & $1379 \_45 \mathrm{mbsf}$ & $\mathrm{k} \_$Archaea (UID2) & Bathyarchaeota & 49.54 & partial & 3.4 & 28.57 & 4 & 191 & 40 & 0 & 21.23 \\
\hline CR_31 & $137945 \mathrm{mbsf}$ & $\begin{array}{c}\mathrm{p} \text { _Euryarchaeota } \\
\text { (UID4) }\end{array}$ & Hadesarchaea & 31.55 & partial & 0.64 & 33.33 & 3.75 & 176 & 40.2 & 0 & 25.74 \\
\hline
\end{tabular}

